\title{
ANÁLISE DE SENSIBILIDADE NO PROCESSAMENTO DE DADOS DE EXPERIMENTOS DE PERFIL INSTANTÂNE $O^{(1)}$
}

\author{
Q. de J ONG van LIER $\mathbf{R}^{(2)}$
}

\begin{abstract}
RESUMO
Dentre as propriedades físicas do solo, a condutividade hidráulica é uma das mais importantes, quando se estudam fenômenos que estão ligados ao movimento da água no solo. 0 método para determinar a função condutividade hidráulica versus umidade ou função $K(\theta)$ de um solo, denominada método de perfil instantâneo, tem como maior vantagem o fato de se basear em medições diretas no campo, sem necessidade de aguardar o estabelecimento de um equilíbrio dinâmico. No caso de experimentos desse tipo, não se conhecem estudos sobre sua sensibilidade em relação a erros nos dados experimentais; tampouco se conhece a influência que uma observação experimental a mais ou a menos possa ter sobre a parametrização da função $K(\theta)$. O presente trabalho visou analisar a sensibilidade do método de perfil instantâneo a variações nas observações realizadas in situ. Utilizaram-se dados de dois experimentos de perfil instantâneo realizados no campus "Luiz de Queiroz", da Universidade de São Paulo, em Piracicaba (SP). Por meio de um algoritmo computacional para a análise dos dados experimentais, verificou-se a sensibilidade do resultado do cálculo dos parâmetros da função $K(\theta)$ para variações nos valores de entrada. Foi analisado o efeito das seguintes alterações no conjunto original de dados: retirada de uma leitura completa do conjunto de dados; retirada de leituras finais; erro no coeficiente angular de calibração do TDR; erro no intercepto da curva de cali bração do TDR; erro em uma das leituras do TDR. Pelos resultados do levantamento, conclui u-se que o método do perfil instantâneo, conjuntamente com a rotina computacional utilizada para processar os valores observados no campo, apresenta alta sensibilidade a pequenas incertezas no ajuste de funções aos valores da umidade versus tempo; étambém muito sensível à leitura do tempo zero, que, portanto, deveria ser desconsi derada. E rros ou incertezas nos ajustes dos valores do potencial total versus a profundidade têm menor influência no
\end{abstract}

(1) Parte da Tese de Livre-Docência do autor, apresentada à Escola Superior de Agricultura "Luiz de Queiroz" - ESALQ/USP, na disciplina "Física do Meio Ambiente". Dados obtidos com auxílio da Fapesp. Recebido para publicação em abril de 2001 e aprovado em julho de 2002.

(2) Professor do Departamento de Ciências Exatas, Escola Superior de Agricultura "Luiz de Queiroz" - ESALQ/USP. Caixa Postal 9, CEP 13418-900 Piracicaba (SP). Bolsista do CNPq. 
resultado final. Se houver comportamento não estritamente exponencial entre condutividade hidráulica e umidade, a sensi bilidade do método para pequenas alterações no conjunto de dados de entrada aumenta. Respectivos erros no coeficiente angular e no intercepto de um TDR com equação de cali bração linear alteram os respectivos coeficiente angular $(\gamma)$ ou intercepto $\left(\ln \left(K^{*}\right)\right)$ da relação entre condutividade hidráulica e umidade, quando o potencial matricial é obtido por tensiometria.

Termos de indexação: condutividade hidráulica; método de campo.

\title{
SUMMARY: SENSITIVITY ANALYSIS OF A COMPUTER ALGORITHM IN DATA PROCESSING FROM INSTANTANEOUS PROFILE EXPERIMENTS
}

\begin{abstract}
Among physical soil properties, hydraulic conductivity is oneof themost important for studies which involve soil water movement. Establishing a relation between hydraulic conductivity and soil water content $(K(\theta)$ function) is essential for such studies. Themethod to determine the $K(\theta)$ function in a soil, called "instantaneous profile method", has one main advantage: it is based on direct field measurements with no need of awaiting the establishment of steady-state conditions. For this kind of experiment, no studies could be found about sensitivity to errors in experimental data or what influence one experimental observation moreor less would have on the parameterization of theK $(\theta)$ function. Based on two instantaneous profile experiments (carried out on the 'Luiz de Queiroz' campus of the Universidade de São Paul o in Piracicaba, State of São Paul o, Brazil), this study tried to anal yzethesensitivity of theinstantaneous profilemethod to variations in field observations, using a computer al gorithm for the analysis of the experimental data. The effect of the fol lowing modifications in the original data set were checked: removal of one observation; removal of final observations; error in thein theangular coefficient of theTDR calibration; error in theintercept of theTDR calibration; error in oneof theTDR readings. Results show that theinstantaneous profilemethod, together with theapplied computer al gorithm, has a high sensitivity to small uncertainties in the fitting of functions to soil moisture versus time. As it is also very sensitiveto thezero timereading, which, therefore, should best not be considered. Errors or uncertainties in fitting soil water potential to depth proved to havea smaller influence on thefinal result. A not strictly exponential behavior between hydraulic conductivity and soil moisture increases the method's sensitivity to small errors. Errors in theangular coefficient or intercept of a TDR with linear cali bration equation only affect the angular coefficient $(\gamma)$ or intercept $\left(\operatorname{In}\left(K^{*}\right)\right)$ of the $K(\theta)$ function, respectively, when thematrix potential is determined by tensiometry.
\end{abstract}

Index terms: hydraulic conductivity; field method.

\section{INTRODUÇÃO}

Dentre as propriedades físicas do solo, a condutividade hidráulica é uma das mais importantes quando se estudam fenômenos que estão ligados ao movimento da água no solo. O método para determinar a função condutividade hidráulica versus umidade $(K(\theta))$ de um solo, denominado método de perfil instantâneo, que já se encontra na literatura da década de 60 (Watson, 1966), foi aperfeiçoado e simplificado por Hillel et al. (1972) e Libardi et al. (1980). Esse método, no qual se fazem observações da umidade do solo e, ou, do potencial matricial da água nele contida em algumas profundidades e ao longo do tempo, para poder chegar a uma estimativa dos parâmetros empíricos da função $K(\theta)$, é utilizado com freqüência, citandose, por exemplo, dentre os trabalhos dos últimos anos, os de Marion et al. (1994), Prevedello et al. (1995), Comegna et al. (1996), Andreu et al. (1997), Reichardt et al . (1998), Simunek et al. (1998) eJ ong van Lier \& Libardi (1999).

A maior vantagem do método está no fato de basear-se em medições diretas no campo, sem necessidade de aguardar o estabel ecimento de um equilíbrio dinâmico ("steady state") e permitindo a análise de uma faixa grande de umidades. Comparando valores obtidos por diferentes métodos de campo e de laboratório, Marion et al. (1994) concluíram que os resultados obtidos pelo método 
do perfil instantâneo in situ são os mais consistentes. Por isso esse método é freqüentemente considerado o padrão.

Pel o fato de o método requerer al gumas semanas até meses de trabal ho de campo, especial mente em sol os com camadas adensadas ou compactadas, onde o processo de drenagem é muito lento, a informação disponível sobre a variabilidade confiabilidade das medições é escassa. As conseqüências disso agravam-se, uma vez quea condutividade hidráulica é uma propriedade do solo que apresenta variabilidadeespacial muitoalta (Warrick \& Nielsen, 1980; Vieira et al., 1981; Bouma et al., 1989; Logsdon \& J aynes, 1996). Numa análise de um experimento de perfil instantâneo com doze pontos de observação, J ong van Lier \& Libardi (1999) encontraram coeficientes de variação muito al tos dos parâmetros empíricos que descrevem a relação entre condutividade hidráulica e umidade.

Além do trabal ho, outra desvantagem do método do perfil instantâneo está na análise gráfica dos gradientes da umidade no tempo e do potencial em profundidade, muitas vezes feita visualmente e, portanto, subjetivamente. Essa desvantagem pode ser minimizada por técnicas computacionais de regressão, como as utilizadas por J ong van Lier \& Libardi (1999). Esses autores calcularam os gradientes da umidade do solo versus o tempo e do potencial total da água no solo versus a profundidade, necessários ao método, conforme detalhado mais adiante, pela derivada de funções ajustadas por regressãolinear, evitando subjetividades na análise dos resultados e tornando os resultados repetíveis.

A calibração de equipamentos de medição da umi dade do solo consiste em estabel ecer uma relação entre observações da leitura do equipamento em questão e um método-padrão, no caso a umi dade do solo determinada pelo método gravimétrico. Erros de calibração foram discutidos por vários autores, destacando-se Haverkamp et al. (1984) e Chanasyk $\&$ Naeth (1996), para a sonda de nêutrons, e Dasberg \& Hopmans (1992), Hook \& Livingston (1996) e Ponizovsky et al. (1999), para o TDR. Haverkamp et al. (1984) mostraram que, no erro total de uma observação de umidade com sonda de nêutrons, o componente relativo à cal ibracao da sonda éo maior. Além disso, demonstraram que a forma de estimar a armazenagem a partir de dados de umidade afeta drasticamente o resultado. No caso da análise da umi dade do sol o por meio deTDR, esta também está sujeita a erros de calibração. Embora exista a "equação universal de calibração" de Topp et al . (1980), trabal hos posteriores, como os de Dasberg \& Hopmans (1992) e de Ponizovsky et al. (1999), indicaram a dependência dessa calibração da natureza da matriz do sol o (granulometria, natureza dos minerais, teor de matéria orgânica).

A análise de sensibilidade, em que a incerteza nos resultados de saída é atribuída à incerteza dos parâmetros deentrada de um model o, éum elemento de verificação para garantir que a resposta de uma rotina computacional atende à expectativa (Saltelli \& Scott, 1997). Em model os simples, a análise de sensibilidade podeser realizada analiticamente. No entanto, numa análise de sensibilidade de um al goritmo mais complicado, mecanístico, o método é empírico, alterando cada parâmetro de entrada ligeiramente para verificar o efeito no resultado de saída. A alteração relativa nos resultados do model o resulta em coeficientes de sensibilidade. Como os parâmetros são variados um por um, o método baseia-se na suposição de que não há relação entre os diferentes parấmetros do algoritmo. Essa suposição é sati sfeita quando a faixa de variação do parâmetro é suficientemente estreita (Drechsler, 1998).

Para experimentos do tipo "perfil instantâneo", não se conhecem na literatura estudos sobre sua sensibilidade em relação a erros nos dados experimentais. No presente trabalho, propôs-se analisar essa sensibilidade, objetivando fornecer subsídios para uma análise mais criteriosa de resultados obtidos por esse tipo de experimento.

\section{MATERIAL E MÉTODOS}

\section{Fundamentos teóricos do método do perfil instantâneo}

No método do perfil instantâneo, procura-se uma solução para a equação de Richards:

$$
\frac{\partial \theta}{\partial t}=\frac{\partial}{\partial z}\left(K(\theta) \frac{\partial \psi_{t}}{\partial z}\right)
$$

em que $\theta\left(\mathrm{m}^{3} \mathrm{~m}^{-3}\right)$ é a umidade volumétrica, $\mathrm{t}(\mathrm{h})$ éo tempo, $\mathrm{K}\left(\mathrm{m}^{2} \mathrm{~h}^{-1} \mathrm{kPa}^{-1}\right)$ éa condutividade hidráulica, $\psi_{\mathrm{t}}(\mathrm{kPa})$ é o potencial total da água e $\mathrm{z}(\mathrm{m})$ é a coordenada vertical de posição. Relacionando a equação de Richards com a profundidadez, entre os limites $z=0$ e $z=z$, com as seguintes condições de contorno:

$$
\begin{aligned}
& z=0 ; t>0 ; q=0 \\
& z=z ; t>0 ; q=q_{z}
\end{aligned}
$$

obtém-se:

$$
\int_{0}^{\mathrm{z}} \frac{\partial \theta}{\partial \mathrm{t}} d z=\left.K(\theta) \frac{\partial \psi_{\mathrm{t}}}{\partial \mathrm{z}}\right|_{\mathrm{z}}=q_{\mathrm{z}}
$$

ou, rearranjando,

$$
\left.K(\theta)\right|_{\mathrm{z}}=\frac{\int_{0}^{\mathrm{z}} \frac{\partial \theta}{\partial t} d z}{\left.\frac{\partial \psi_{t}}{\partial z}\right|_{z}}=\frac{\frac{\partial h_{z}}{\partial t}}{\left.\frac{\partial \psi_{t}}{\partial z}\right|_{z}}
$$


em que $h_{z}\left(m^{3} m^{-2}\right)$ é a armazenagem de água no solo entrea superfície $(z=0)$ ea profundidade $z$. Por meio de medidas simultâneas de umidade e de potencial total ao longo do perfil de solo durante a redistribuição da água, determinam-se a variação de armazenagem $\mathrm{h}_{\mathrm{z}}$ com o tempo t e o gradiente de $\psi_{\mathrm{t}}$ ao longo de $z$ que aparecem na equação 3 e, então, o val or de $\mathrm{K}(\theta)$ para aquela umidade.

Freqüentemente, os valores de $K(\theta)$ apresentam uma relação exponencial com a umi dade em al guma faixa de umi dade, de maneira que a função $K(\theta)$ pode ser expressa pela equação

$$
K(\theta)=K^{*} \cdot e^{\gamma \theta}
$$

sendo que $\gamma$ é uma constante adimensional e $K^{*}\left(\mathrm{~m}^{2} \mathrm{~h}^{-1}\right.$ $\mathrm{kPa}^{-1}$ ) o val or hipotético da condutividade hidráulica quando a umidade do sol o equivale e zero.

\section{Métodos de campo}

Visando analisar a sensibilidade do método do perfil instantâneo a variações nas observações realizadas, utilizaram-se dados de dois experimentos de drenagem, realizados, durante os meses de junho e julho de 2000, em dois locais do campus "Luiz de Queiroz", da Universidade de São Paulo, em Piracicaba (SP).

No primeiro local (local P), em um Latossolo Vermelho-Amarelo álico horizonte A moderado textura média, localizado em Piracicaba (SP), a umidade foi monitorada durante o processo de drenagem por um TDR segmentado (modelo Moisture Point ${ }^{\mathrm{TM}}$, tipo "H", fabricado por E.S.I Environmental Sensors Inc.) com sensores nas profundidades de $0-0,15,0,15-0,30,0,30-0,45,0,45$ 0,60 e 0,60-0,75 m. O potencial matricial foi medido por tensiometria nas mesmas profundidades. Em decorrência de problemas com ouso do equi pamento deTDR na profundidade de 0-0,15 m utilizaram-se para essa profundidade os valores de 0,15-0,30 m.

No segundo local (local A), um experimento de drenagem foi realizado no canal de um terraço de infiltração na fazenda experimental "Areão" da ESALQ/USP, em Piracicaba (SP). Leituras deTDR (SoilMoisture Equipment Corp., modelo TRASE ${ }^{\mathrm{TM}}$, tipo "buriable"), instalados horizontalmente nas profundidades de $0,05,0,10,0,20,0,40,0,60$ e0,80 m, foram registradas automatizadamente de meia em meia hora num Latossolo Vermel ho-Escuro textura argilosa, com densidades em torno de $1.100 \mathrm{~kg} \mathrm{~m}^{-3}$ no $A_{p}$ e ao redor de $1.000 \mathrm{~kg} \mathrm{~m}^{-3}$ nas camadas mais profundas.

Antes de iniciar as observações, os solos dos dois locais foram umedecidos. No local $P$, isso foi feito por aspersão e, no local A, por inundação, atingindo uma condição bastante úmida e de fluxo descendente. Em seguida, a superfície do solo foi coberta com uma lona plástica, evitando a infiltração ou evaporação de água na superfície do solo, satisfazendo a primeira condição de contorno do método. A partir desse momento $(t=0)$, a umidade do solo nas profundidades de instalação dos equipamentos foi monitorada por meio de leituras com o TDR em ambos os locais, A e P. No local P, utilizaram-se também tensiômetros para observar os valores do potencial matricial nas mesmas profundidades. O potencial matricial nolocal A não foi medido, mas, posteriormente, foi estimado com base nas curvas de retenção.

Nolocal A, foram feitas duas leituras automáticas por hora. Para fins deste estudo, selecionaram-se, desse conjunto grande de observações, quatro leituras do primeiro dia (de seis em seis horas) e mais 21 leituras das semanas seguintes, com interval os cada vez mai ores ( 12 horas, inicial mente, até 80 horas, no fim), até compl etar três semanas de monitoramento da drenagem. Nolocal $\mathrm{P}$, a primeira leitura foi realizada no tempo 0 , a segunda aproximadamente seis horas depois e a terceira 24 horas depois. A seguir, mantiveram-se intervalos de dois a três dias entreas leituras, atéa $25^{a}$ leitura, 57 dias depois do início do experimento, quando o processo já estava muito lento (Figura 1 ).

A calibração entre a leitura doTDR $\left(K_{a}\right)$ em ns e a umidade do solo $(\theta)$ foi realizada por meio de observações simultâneas da leitura do TDR e da umidade gravimétrica. Para o local A, essa calibração foi feita em pequenas colunas em laboratório, e os resultados ajustaram-se bem à equação

$$
\theta=a+\frac{b}{\sqrt{K_{a}}}
$$

em que a e b (ns ${ }^{0,5}$ ) são os parâmetros empíricos de calibração. No local P, as observações foram feitas no campo em algumas datas logo após a execução do experimento, em diferentes pontos de medição do experimento. Os valores ajustaram-se bem à equação de primeiro grau:

$$
\theta=a+b K_{a}
$$

sendo a e b (ns $\left.{ }^{-1}\right)$ os parâmetros empíricos de calibração.

Nolocal A, ondenãoforam utilizados tensiômetros, elaboraram-se curvas de retenção para cada profundidade, com vistas em estimar o potencial matricial de acordo com as umidades observadas. Duas amostras indeformadas por profundidade foram analisadas gravimetricamente, quando submetidas, em laboratório, a diferentes tensões $(0,5$; 1,0; 2,0; 6, 1 e 10,2 kPa, em caixa de areia; 10; 20; 34; 46; 102; 306 e $510 \mathrm{kPa}$, em câmara de pressão). Os valores assim obtidos, juntamente com o valor referente à tensão de 0 kPa (saturação), calculado com base na densidade do solo, foram ajustados à equação deVan Genuchten (1980), estimando-seseus parâmetros por regressão não-linear iterativa. 


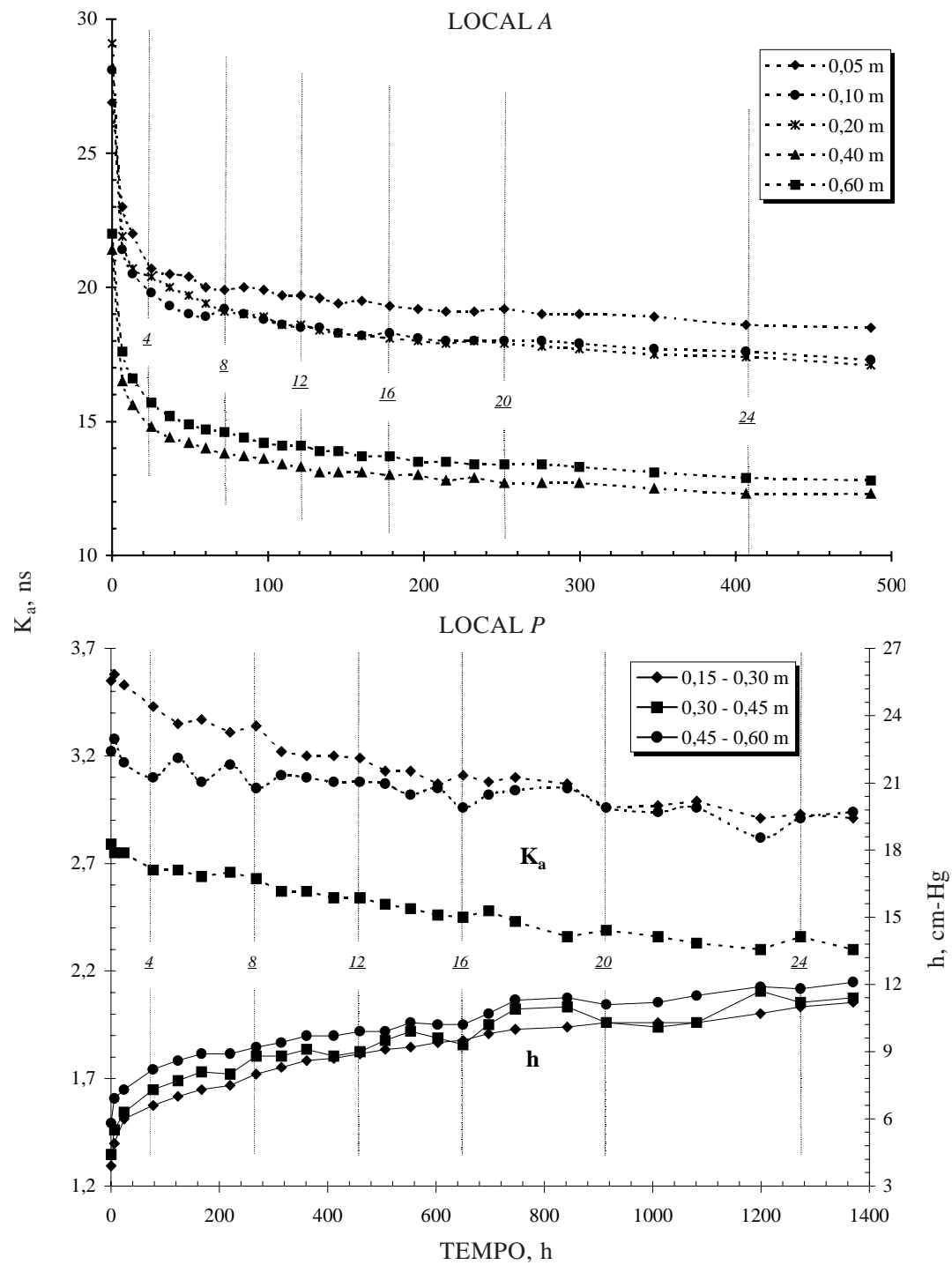

Figura 1. Leituras do TDR (Ka, ns) e dos tensiômetros $(\mathrm{h}, \mathrm{cm}-\mathrm{Hg})$ ao longo do tempo $(\mathrm{h})$ nas profundidades de observação nos locais A e P. (os números sublinhados no gráfico referem-se ao número seqüencial da leitura).

\section{Análise computacional}

Visando obter os parâmetros empíricos da equação 4, as leituras de cada ponto de observação foram analisadas, utilizando-se um programa de computador baseado em procedimentos descritos adiante. Da mesma forma, obtiveram-se esses parâmetros fazendo-se pequenas alterações nos conjuntos de dados originais para verificar a sensibilidade do al goritmo, conforme descrito mais adiante.

A partir das leituras doTDR ( $\left.\mathrm{K}_{a}, \mathrm{~ns}\right)$, obtiveramse as umidades pelas respectivas equações de calibração dos equipamentos de TDR (equação 5a, para o local $A$; equação $5 b$, para o local P).

Os valores da umidade do solo em função do tempo $(t, h)$ foram ajustados, para cada profundidade, a uma das equações $6 a$ a $6 \mathrm{c}$ :

$$
\begin{gathered}
\theta=n_{z} t^{m_{z}} \\
\theta=n_{z}+m_{z} t^{0,5} \\
\theta=n_{z}+m_{z} \ln t
\end{gathered}
$$

sendo $m_{z}$ e $n_{z}$ os coeficientes de ajuste da equação. Como critério para decidir qual das equações $(6 a, 6 b$ ou 6c) utilizar, usou-se o do coeficiente de determinação $R^{2}$ : a equação que apresentou maior $R^{2}$ com os dados de determinada profundidade foi utilizada para aquela profundidade. Pela derivada da respectiva equação $6 a, 6 b$ ou $6 c$, estimou-se, para cada tempo intermediário entre dois tempos de observação, o termo $\partial \mathrm{h}_{\mathrm{z}} / \partial \mathrm{t}$ da equação 3 , pela expressão

$$
\frac{\partial h_{z}}{\partial t}=\left.\sum_{i=1}^{z} z_{i} \frac{\partial \theta}{\partial t}\right|_{i}
$$


em que $z_{\mathrm{i}}(\mathrm{m})$ éa espessura da camada i. O potencial matricial de cada profundidade e tempo foi obtido pela leitura dos tensiômetros (local P) ou pela equação deVan Genuchten (local A). Para os tempos intermediários entre as leituras, calcularam-se os potenciais matriciais como val or médio entre leitura anterior e posterior e, somando-se o valor do potencial gravitacional, obteve-se o potencial total da água no solo $\left(\psi_{\mathrm{t}}, \mathrm{kPa}\right)$ em cada tempo e profundidade, que foi ajustado, para cada tempo, à equação

$$
\psi_{t}=p_{t}+q_{t} z+r_{t} z^{2}
$$

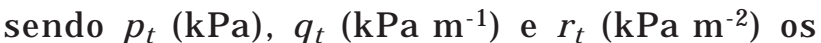
coeficientes de ajuste da equação. Pela equação 7 e a derivada da 8 calcularam-se, para cada t e z, os dois elementos do membro direito da equação 3 , obtendo o valor de $K(\theta)$ para a umidade correspondente. A partir do conjunto de pares de dados $(K, \theta)$ assim obtidos, estimaram-se os parâmetros $K^{*}$ e $\gamma$ da equação 4 por meio deregressão linear, tomando como variável dependente o $\ln [K(\theta)]$ e como variável independente $\theta$.

\section{Cenários para os testes de sensibilidade}

Analisou-se a sensibilidade do resultado do cálculo dos parâmetros $K^{*}$ e $\gamma$ da equação 4 para variações nos valores de entrada para os locais $A$ e $P$. No local A, compararam-se os resultados para a profundidade de $0,20 \mathrm{~m}$; no local $\mathrm{P}$, a profundidade analisada foi a de 0,30 a 0,45 m. Foi analisado o efeito das seguintes alterações no conjunto original de dados:

1. Retirada de uma leitura completa do conjunto de dados

2. Retirada de leituras finais

3. Erro no coeficiente angular de calibração do TDR

4. Erro no intercepto da curva de calibração do TDR

5. Erro em uma das leituras doTDR
Os val ores dos parâmetros $\gamma$ e $\ln \left(\mathrm{K}^{*}\right)$ obtidos com os dados al terados foram comparados com os obtidos com o conjunto intacto de dados $\left(\gamma_{0}\right.$ e $\left.\ln \left(\mathrm{K}^{*}\right)_{0}\right)$ por meio dos quocientes $\gamma / \gamma_{0}$ e $\left.\ln \left(K^{*}\right) / \operatorname{In}\left(K^{*}\right)_{0}\right)$, respectivamente. Quocientes mais próximos da unidade indicam maior semelhança entre o resultado com o conjunto de dados original e com o conjunto alterado, e viceversa. Para uma análise estatística mais completa, haveria necessidade de repetições, não-disponíveis para o presente estudo.

\section{RESULTADOS E DISCUSSÃO}

A análise computacional mostrou ajustes bons à equação 6 ( $R^{2}>0,9$ em quase todos os casos) e à equação 8 ( $R^{2}>0,9$ em todos os casos). O ajuste à equação 4 resul tou em $R^{2}$ entre 0,8 e 0,9 , para ol ocal $P$, e maiores que 0,99 , para o local $A$. O menor $R^{2}$ no local $P$ deve-se, principalmente, a um comportamento não estritamente exponencial, especialmente na parte mais úmida, como mostra a figura 2. Um comportamento não-exponencial como o da figura 2 foi encontrado também por outros autores, como Prevedello et al. (1995) e Klein (1998). Considerando o grau de desvio da curva exponencial, pode-se decidir pela divisão da faixa de umidades em faixas menores, onde o comportamento seaproxima mel hor da curva exponencial.

Nolocal A, foram determinados $\left(\mathrm{K}^{*}\right)_{0}=4,61 \cdot 10^{-33} \mathrm{~m}^{2}$ $\mathrm{GPa}^{-1} \mathrm{~S}^{-1}$ e $\gamma_{0}=173,8$; no local $\mathrm{P}$, os valores desses parâmetros foram $\left(\mathrm{K}^{*}\right)_{0}=6,88.10^{-8} \mathrm{~m}^{2} \mathrm{GPa}^{-1} \mathrm{~s}^{-1} \mathrm{e}$ $\gamma_{0}=44,25$. Os valores de $K^{*}$, a condutividade hidráulica do solo quando sua umidade for zero, devem ser considerados como meros parâmetros empíricos sem significado físico, uma vez quea faixa de umidade avaliada $\left(0,19-0,25 \mathrm{~m}^{3} \mathrm{~m}^{-3}\right.$ - local $P ; 0,35-$ $0,39 \mathrm{~m}^{3} \mathrm{~m}^{-3}$ - local A) nãoinclui enem chega próximo do valor zero.

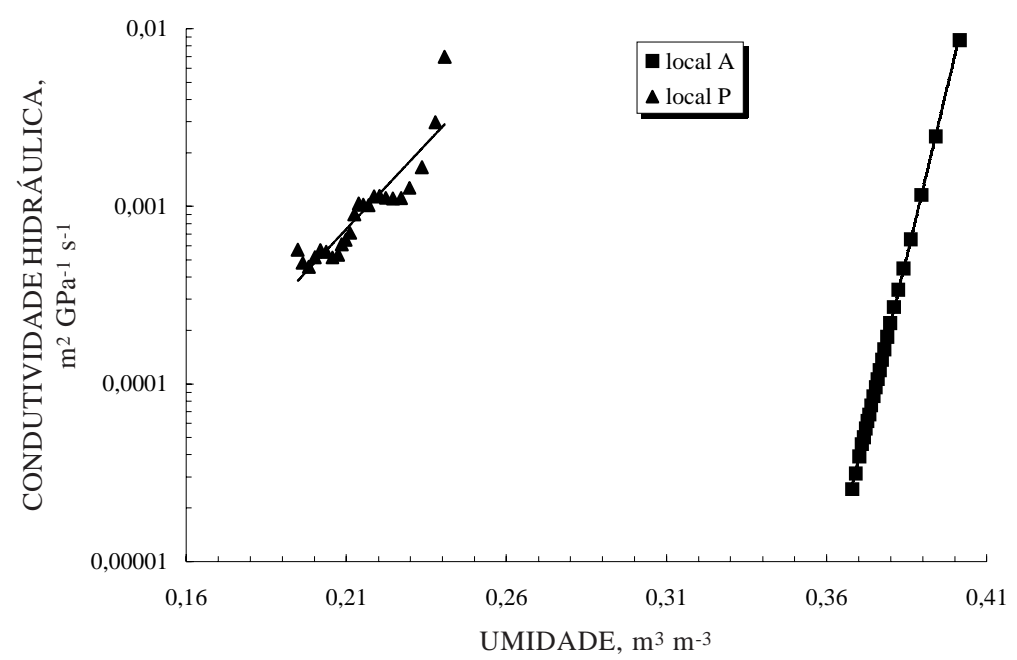

Figura 2. Condutividade hidráulica versus umidade volumétrica para o local $A(0,20 \mathrm{~m})$ e para o local $P$ $(0,30-0,45 \mathrm{~m})$. 
Retirada de uma leitura completa do conjunto de dados

Observa-se, na figura 3, que o método de cálculo em geral não tem grande sensibilidade à exclusão de uma das leituras: os valores de $\gamma / \gamma_{0}$ (ordenada esquerda) e de In $\mathrm{K}^{*} / \mathrm{In} \mathrm{K}^{*}{ }_{0}$ (ordenada direita) mantêm-se em torno de 1,0. No entanto, nota-se um comportamento diferente quando se el imina um dos primeiros pontos. Nesses casos, o resultado de cálculo altera-se significativamente, podendo os parâmetros ser subestimados em até $20 \%$. A explicação para este fato está ilustrada, para o local A, na figura 4, onde se observa, na parte (a), o gráfico dos valores de $\theta$ versus t em escala logarítmica. A exclusão do primeiro ponto $(t=0$, representado na escala logarítmica em $t=0,01 \mathrm{~h}$ ) leva ao ajuste diferente da equação $6 c$, como representado na mesma figura. I sto porque o primeiro ponto tem um peso grande na regressão por estar afastado dos outros pontos na escala logarítmica. Em conseqüência do ajuste diferenciado, obtêm-se valores de $d \theta / d t$ diferentes (parte (b) da figura 4), que resultam em estimativas de condutividade hidráulica diferentes (parte (c) da figura 4), com conseqüentes valores diferentes dos parâmetros $\gamma$ e In $K^{*}$ da equação 4.

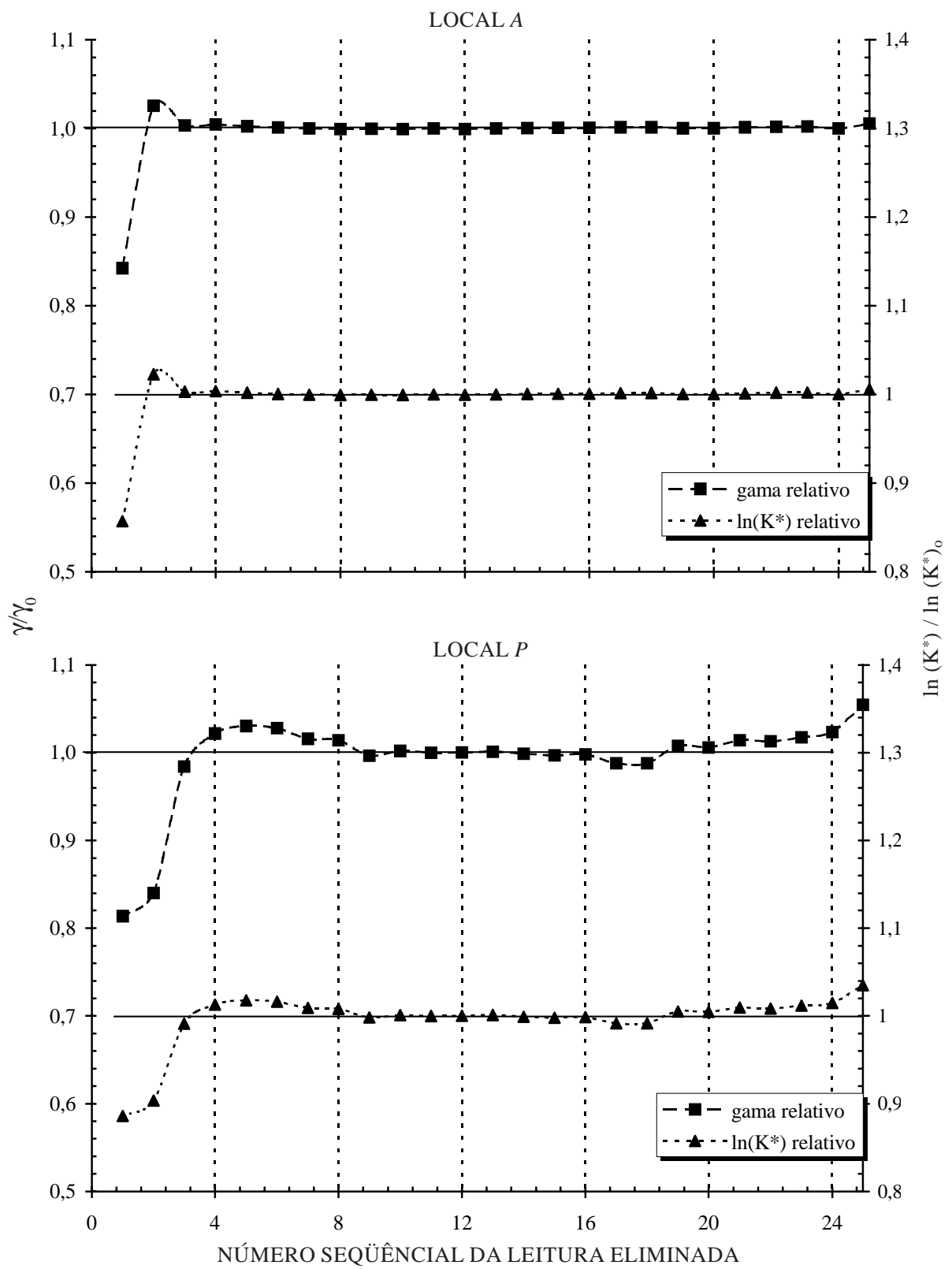

Figura 3. Valores de $\gamma$ e $\ln \left(K^{*}\right)$, obtidos eliminando-se uma por uma cada uma das $\mathbf{2 5}$ leituras realizadas nos locais A e $P$, relativos, respectivamente, aos valores de $\gamma_{0}$ e $\ln \left(K^{*}\right)_{0}$, obtidos do conjunto completo de dados. 

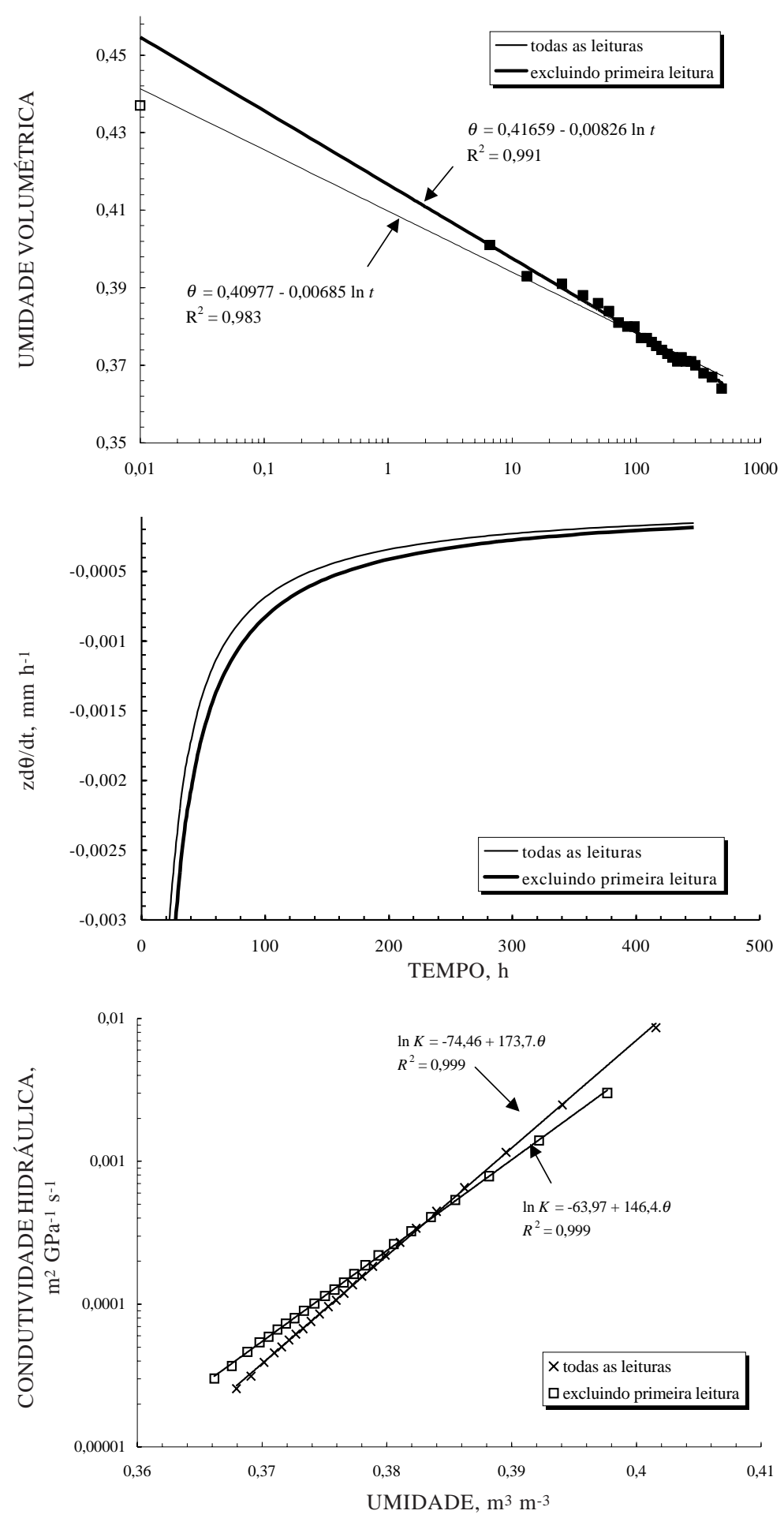

Figura 4. Seqüência exemplar mostrando como, no local A, a exclusão da primeira observação leva a (a) um ajuste diferente da relação entre a umidade e o tempo; (b) valores diferentes para z.d $\theta / d t$ (variação da armazenagem) ao longo do tempo e (c) um resultado diferente para a relação $\mathrm{K}-\boldsymbol{\theta}$.

Ainda observando a figura 3, nota-se que o comportamento dos valores de $\gamma / \gamma_{0}$ é muito semel hante ao dos val ores de In $\mathrm{K}^{*} / \mathrm{In} \mathrm{K}^{*}{ }_{0}$ enquanto, pela lógica, um $\gamma$ maior levaria a um $\ln \left(\mathrm{K}^{*}\right)$ menor. Para interpretar o significado desse fato, deve-se levar em consi deração que os val ores de $\gamma$ são sempre positivos, enquanto o In K* é sempre negativo, uma vez que os valores de ${ }^{*}$, na unidade escol hida, estão na ordem de grandeza de $10^{-8}$ (Iocal P) ou 10-33 (local A). Dessa forma, um valor de $\ln K^{*} / \ln K^{*}{ }_{0}$ 
menor que 1 significa que In $\mathrm{K}^{*}$ é menos negativo, ou seja, maior que In $\mathrm{K}^{*}{ }_{0}$. Um valor de $\gamma / \gamma_{0}$ menor que 1 indica que $\gamma$ é menos positivo, ou seja, menor que $\gamma_{0}$. Comotambém fica evidenciado na figura $4 c$, uma diminuiçãono val or de $\gamma$ leva, por extrapolação, a um aumentono valor de $\mathrm{K}^{*}$ e deln $\mathrm{K}^{*}$. Desta forma, explica-se porque um val or de $\gamma / \gamma_{0}$ menor que 1 vem sempre acompanhado de um In $\mathrm{K}^{*} / \mathrm{In} \mathrm{K}{ }_{0}^{*}$ também inferior a 1, e vice-versa.

\section{Retirada de leituras finais}

Observando os resultados dos cálculos com retirada de leituras finais para o local $P$, verifica-se, em todos os pontos, que, quanto mais prolongado o experimento, menores setornaram os val ores de $\gamma \mathrm{e}$ menos negativos os de In $\mathrm{K}^{*}$ (Figura 5 ). Analisando este resultado conjuntamente com a figura 2 , verifica-se que parte da explicação está no fato de os pontos relativos às primeiras observações (os de maior umidade) apresentarem um gradiente $(\gamma)$ bem maior que os subseqüentes. Em outras palavras, os parâmetros K e $\theta$ não apresentaram, na faixa avaliada, relação estritamente exponencial. Assim, quanto mais pontos finais são desconsiderados, maior fica o peso relativo dos primeiros pontos e mai or o $\gamma$ estimado. Os val ores finais são atingidos somente com o conjunto praticamente completo de observações, sugerindo até que uma prorrogação do experimento teria alterado o resultado final. A tendência para o local $A$ é semel hante à do local $P$, porém verifica-se que a amplitude dos valores relativos de $\gamma$ e In $\mathrm{K}^{*}$ é bem menor (entre 0,9 e 1,1).

\section{Erro no coeficiente angular de calibração do TDR}

Para o local $P$, um erro no coeficiente angular $b$ $\left(\varepsilon_{b}\right)$ da equação $5 b$, utilizado nos cálculos para esse local, não leva a nenhuma alteração dos valores de $\mathrm{K}^{*}$ estimados, enquanto os val ores de $\gamma$ se modificam proporcionalmente (Figura 6).

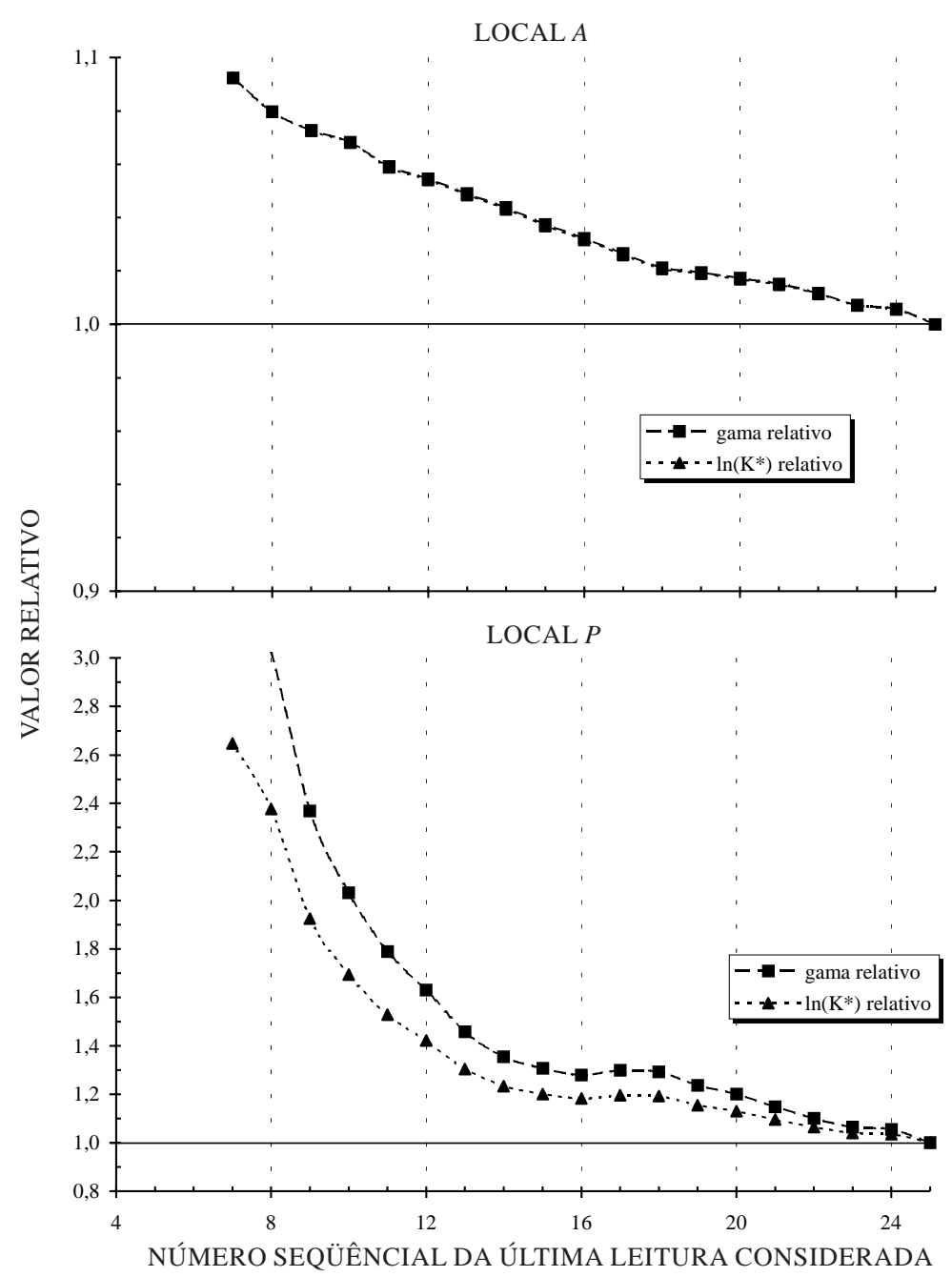

Figura 5. Valores de $\gamma$ e $\ln \left(K^{*}\right)$ relativos, respectivamente, aos valores de $\gamma_{0}$ e $\ln \left(K^{*}\right)_{0}$, obtidos de leituras realizadas até determinado número seqüencial no local $A$ e no local $P$ (no local $A$, as duas linhas estão sobrepostas). 


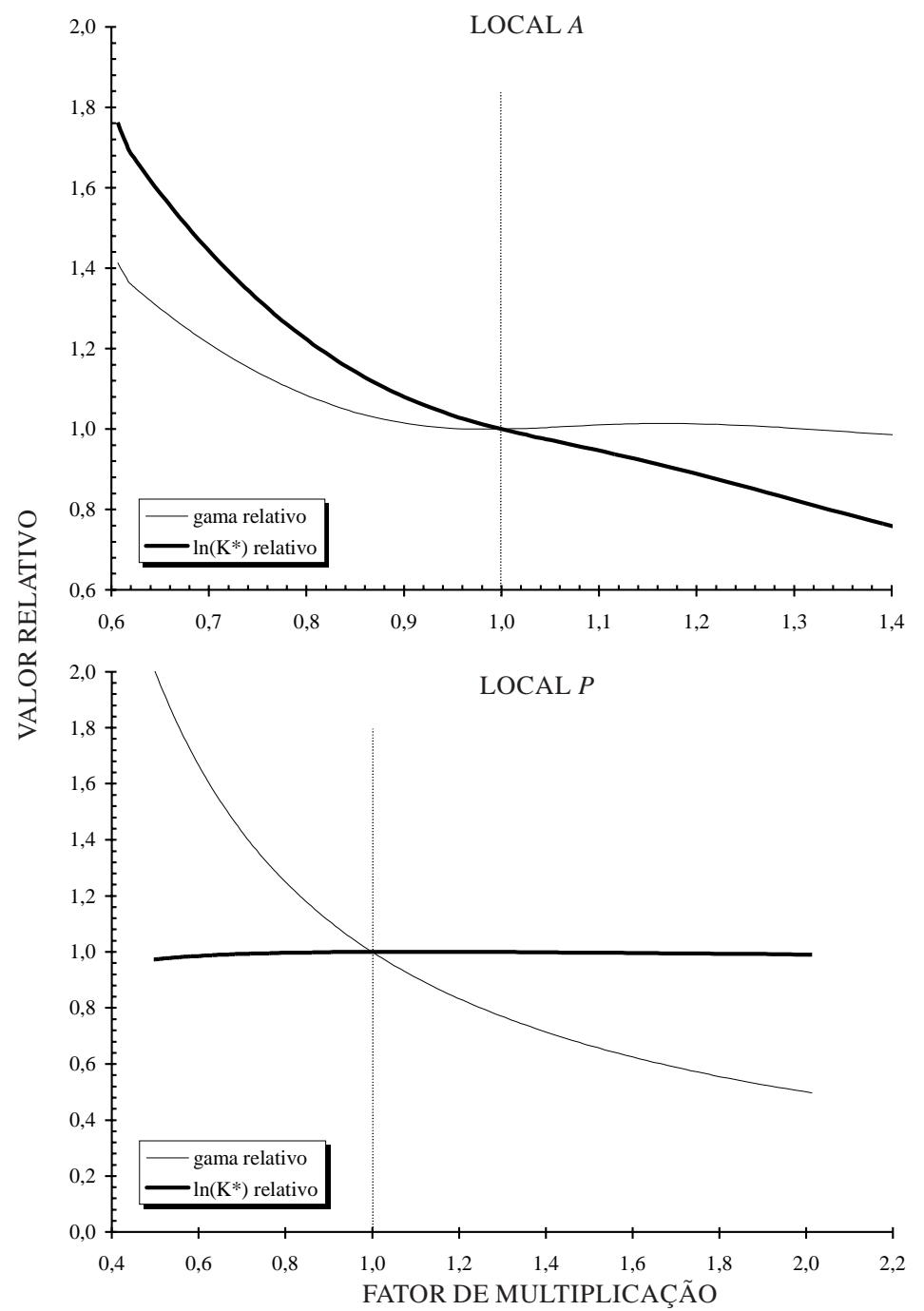

Figura 6. Valores de $\gamma$ e $\ln \left(K^{*}\right)$ para o local $A(0,20 \mathrm{~m})$ e local $P(0,30-0,45 \mathrm{~m})$, obtidos da indução de um erro nos coeficientes angulares de calibração do TDR de todas as profundidades, multiplicando-os pelo fator na abscissa, relativos, respectivamente, aos valores de $\gamma_{0} e \ln \left(K^{*}\right)_{0}$, obtidos do conjunto intacto de dados.

A explicação deste resultado está no fato de um erro no coeficienteangular b da equação $5 b$ resultar numerro $\left(\varepsilon_{\theta}\right)$ na umidadeestimada ( $\left.\hat{\theta}\right)$ por essa equação:

$$
\begin{gathered}
\quad \theta=a+b K_{a} \\
\hat{\theta}=a+\left(b+\varepsilon_{b}\right) K_{a}=a+b K_{a}+\varepsilon_{b} K_{a} \\
\Rightarrow \hat{\theta}-\theta=\varepsilon_{\theta}=\varepsilon_{b} K_{a}
\end{gathered}
$$

Dessa forma, quanto maior o valor da leitura do $\operatorname{TDR}\left(\mathrm{K}_{\mathrm{a}}\right)$, maior $\varepsilon_{\theta}$. Como os valores de $\mathrm{K}_{\mathrm{a}}$ diminuem ao longo do experimento (Figura 1), os valores de $\varepsilon_{\theta}$ também diminuirão. Nocaso deuma superestimativa do coeficiente angular $\left(\varepsilon_{b}>0\right)$, obtêm-se val ores de umidade levemente mais superestimados no início que no fim do experimento (Figura 7a). Assim, o termo $\partial \mathrm{h}_{\mathrm{z}} / \partial \mathrm{t}$ da equação 3 será, em módulo, maior (Figura $7 b$ ), enquanto o denominador $\partial \psi_{t} / \partial z$, que depende das leituras dos tensiômetros, ficará inal terado. Como resultado, os valores deK $(\theta)$ serão maiores e tanto maiores quanto for a umidade. No entanto, cada val or de $\mathrm{K}(\theta)$ será referentea um valor de umidade também maior. Esse conjunto de alterações leva a valores de $\gamma$ menores e de $\mathrm{K}^{*}$ inalterados, como se observa também na figura 7c.

Para o local A, onde a equação de calibração do TDR foi a equação 5 a e onde os valores dos potenciais, matricial etotal, são calculados em função da umi dade medida pel oTDR, o comportamento dos resultados de cálculo em função de um erro no coeficiente angular da equação de calibração foi diferente (Figura 6). Para esses casos: 


$$
\begin{gathered}
\theta=a+\frac{b}{\sqrt{K_{a}}} \\
\hat{\theta}=a+\frac{b+\varepsilon_{b}}{\sqrt{K_{a}}}=a+\frac{b}{\sqrt{K_{a}}}+\frac{\varepsilon_{b}}{\sqrt{K_{a}}} \\
\Rightarrow \hat{\theta}-\theta=\varepsilon_{\theta}=\frac{\varepsilon_{b}}{\sqrt{K_{a}}}
\end{gathered}
$$

Como os valores de b para essa equação foram sempre negativos, a multiplicação com um fator maior que 1 equivale a um $\varepsilon_{b}<0$ ou seja, a uma subestimativa de $\theta$, tanto menor quanto maior for $\mathrm{Ka}$. Como no local P ocorrem estimativas diferentes da umi dade vol umétrica, com conseqüente al teração na relação $\theta$-t e no termo variação da armazenagem $\left(\partial \mathrm{h}_{\mathrm{z}} / \partial \mathrm{t}\right)$ da equação 3. Nesse caso, no entanto, o denominador daquela equação $\left(\partial \psi_{t} / \partial z\right)$ é também afetado de uma forma que depende do formato (nãolinear) da respectiva curva de retenção. Sendo assim, o resultado final é alterado, tanto no coeficiente angular $\gamma$ como no intercepto In $\mathrm{K}^{*}$, de forma irregular. Nesses casos, na faixa de erro de $-10 \%$ a $+10 \%$ (fatores de multiplicação entre 0,9 e 1,1), aparentemente a subestimativa de b (fator de multiplicação >1) leva a val ores relativos de $\gamma$ eln $\mathrm{K}^{*}$ menores que 1 , indicando subestimativa de $\gamma$ e superestimativa (valores menos negativos) de In $\mathrm{K}^{*}$.

\section{Erro no intercepto da curva de calibração do TDR}

De forma semelhante ao discutido no item anterior, verifica-se que um erro no intercepto a $\left(\varepsilon_{a}\right)$ da equação $5 b$, utilizado para o TDR do local $P$, resultará num erro $\left(\varepsilon_{\theta}\right)$ na estimativa de $\theta(\hat{\theta})$ :
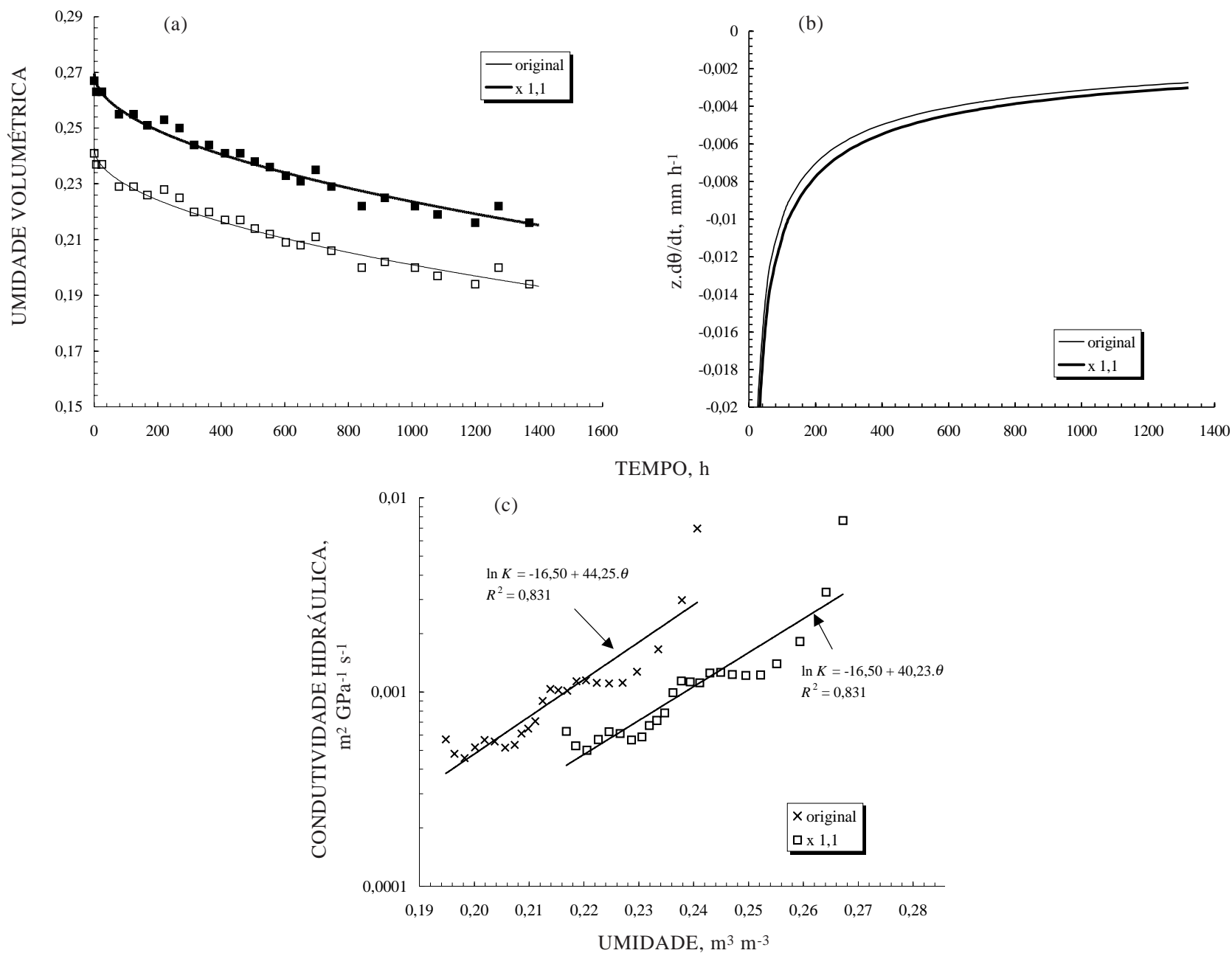

Figura 7. Seqüência exemplar mostrando como, para o local P; 0,30-0,45 m, a superestimativa do coeficiente angular da equação 5b leva a (a) alterações nas umidades calculadas ao longo do tempo; (b) valores ligei ramente diferentes para z.d $/$ /dt (variação da armazenagem) ao longo do tempo e (c) um coefi ciente angular menor para a relação $\mathrm{K}-\theta$. 


$$
\begin{gathered}
\theta=a+b K_{a} \\
\hat{\theta}=\left(a+\varepsilon_{a}\right)+b K_{a} \\
\Rightarrow \hat{\theta}-\theta=\varepsilon_{\theta}=\varepsilon_{a}
\end{gathered}
$$

Anal ogamente, para o local A (equação $6 b$ ):

$$
\begin{gathered}
\theta=a+\frac{b}{\sqrt{K_{a}}} \\
\hat{\theta}=\left(a+\varepsilon_{a}\right)+\frac{b}{\sqrt{K_{a}}} \\
\Rightarrow \hat{\theta}-\theta=\varepsilon_{\theta}=\varepsilon_{a}
\end{gathered}
$$

Dessa forma, em ambos os casos, tem-se um erro constante na estimativa de $\theta$, independentemente do valor deKa. Assim, os valores de d $\theta / d t$ não serão afetados e nem os de $\partial \mathrm{h}_{\mathrm{z}} / \partial \mathrm{t}$.

Para o local $\mathrm{P}$, onde os gradientes de potencial foram medidos independentemente com tensiômetros, encontraram-se valores de condutividade hidráulica iguais, porém estes se referiram a umi dades maiores (para $\varepsilon_{\mathrm{a}}>0$ ) ou menores (para $\varepsilon_{\mathrm{a}}<0$ ), resultando em coeficientes angulares $(\gamma)$ iguais e interceptos (In K*) diferentes. Como os valores de a do local $\mathrm{P}$ até à profundidade em questão foram negativos, um fator de multiplicação maior que 1 corresponde a um $\varepsilon_{\mathrm{a}}<0$ e, conseqüentemente, a um $\varepsilon_{\theta}<0$ (subestimativa das umidades). Como resultado, obtêm-se valores de In $\mathrm{K} *$ menos negativos, com valores relativos conseqüentemente menores que 1 .

Para o local A, os valores de a da equação $5 b$ foram positivos e fatores de multiplicação maiores que 1 resultaram em superestimativa das umi dades. Dessa forma, era esperado para esse local um comportamento contrário ao da figura 8: que um aumento do fator de multiplicação resultasse em aumento de $\ln \mathrm{K}^{*} / \mathrm{In} \mathrm{K}^{*}{ }_{0}$. Verifica-se que, aproximadamente, isso ocorre, porém, nesse caso, o comportamento não élinear como no caso do local $P$ pelo fato de, no local $A$, os gradientes de potencial também serem estimados com base na umidade, como já discutido. Por essa razão, os $\gamma$ estimados sofreram alterações, o que não aconteceu no local P.

\section{Erro em uma das leituras do TDR}

Analisando o efeito de um erro em apenas uma das leituras de TDR, verificou-se, na figura 9, que, nocaso de um erro positivo (leitura registrada maior) em uma das leituras da primeira metade da seqüência de observações, o valor relativo $\gamma / \gamma_{0}$ foi maior que 1 (superestimativa de $\gamma$ ). No entanto, se o mesmo erro ocorrer em uma leitura da última metade da seqüência de observações, o val or rel ativo $\gamma / \gamma_{0}$ será menor que 1 (subestimativa de $\gamma$ ). Em alguma leitura no meio da seqüência (próximo à leitura 12, no local P, e leitura 8, no local A), não há nenhum efeito de uma super ou subestimativa da leitura doTDR.

A explicação destes fatos pode ser encontrada na figura 10, que compara, para o local $P$, as relações qt com os val ores originais e com um erro na $3^{a}$ leitura (parte (a) da figura 10), na 12a leitura (parte (b) da figura 10) e na $22^{\text {a }}$ leitura (parte (c) da figura 10). Na parte (a) dessa figura, observa-se que, em virtude

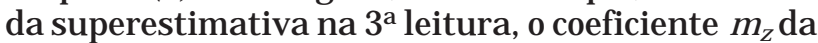
equação de ajuste $6 \mathrm{~b}$ aumentou de $1,33.10^{-3}$ para $1,49.10^{-3}$, resultando em valores mais negativos para

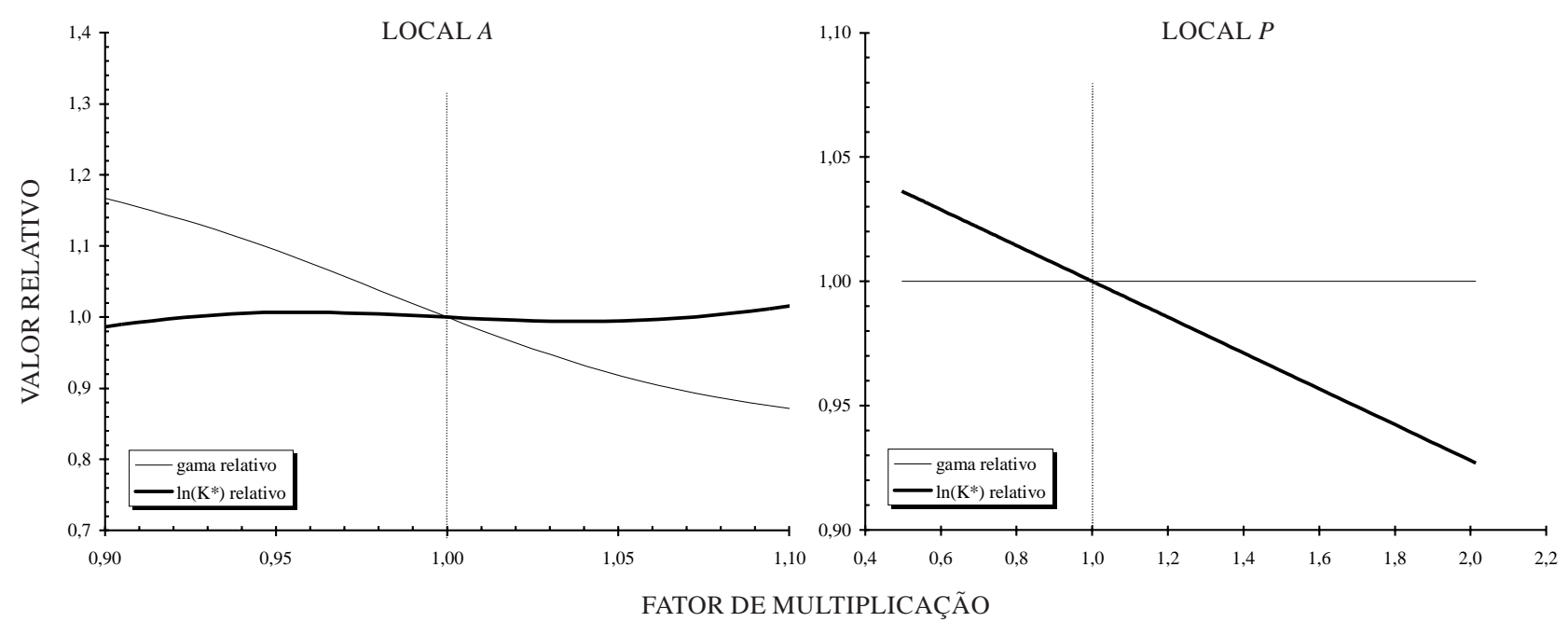

Figura 8. Valores de $\gamma$ e $\ln \left(K^{*}\right)$ para o local $A(0,20 \mathrm{~m})$ e local $P(0,30-0,45 \mathrm{~m})$, obtidos da indução de um erro nos interceptos das curvas de calibração do TDR de todas as profundidades, multiplicando-os pelo fator na abscissa, relativos, respectivamente, aos valores de $\gamma_{0}$ e $\operatorname{In}\left(K^{*}\right)_{0}$, obtidos do conjunto intacto de dados. 


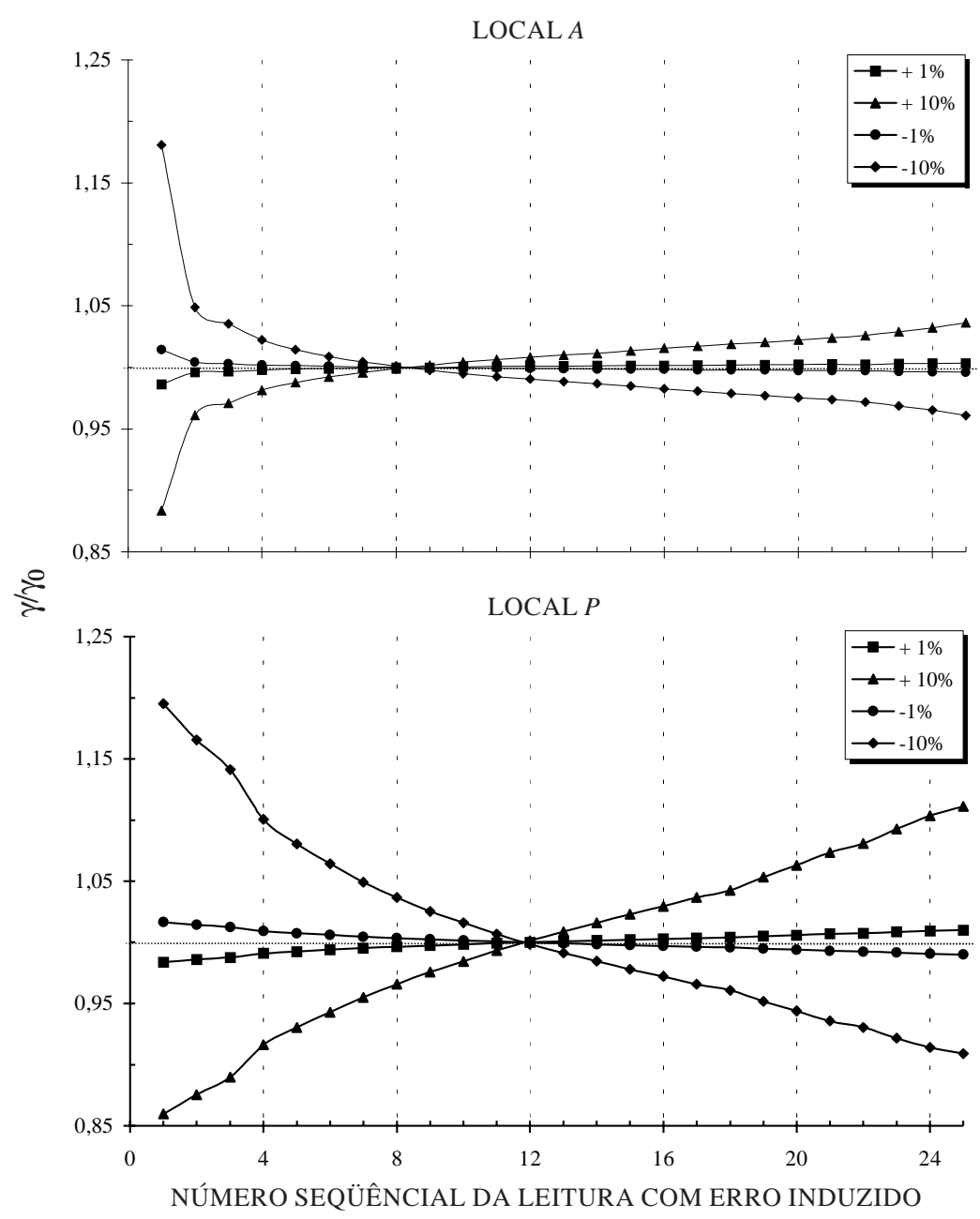

Figura 9. Valores de $\gamma$ para o local $\mathbf{A}(0,20 \mathrm{~m})$ e local $\mathbf{P}(0,30-0,45 \mathrm{~m})$, obtidos da indução de um erro de $-10 \%,-1 \%,+1 \%$ ou $+10 \%$, uma por uma em cada uma das leituras do TDR na mesma profundidade, relativos aos valores de $\gamma_{0}$, obtidos do conjunto intacto de dados.

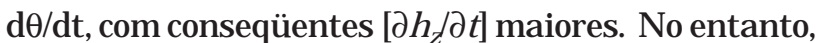
quando o erro ocorre na $12^{\mathrm{a}}$ leitura (parte (b) da figura 10), o coeficiente $m_{z}$ da equação $7 b$ praticamente nãoéafetado (de $1,33 \cdot 10^{-3}$ para $1,32 \cdot 10^{-3}$ ), não alterando tampouco $\mathrm{d} \theta / \mathrm{dt}$ enem $\left[\partial \mathrm{h}_{\mathrm{z}} / \partial \mathrm{t}\right]$. U m erro na leitura 22 (parte (c) da mesma figura) acarreta uma diminuição do coeficiente $m_{z}$ da equação de ajuste $6 \mathrm{~b}$ de $1,33.10^{-3}$ para $1,23.10^{-3}$, resultando em val ores menos negativos para $d \theta / d t$ e menores $\left[\partial \mathrm{h}_{\mathrm{z}} / \partial \mathrm{t}\right]$.

Observa-se também que, com exceção do primeiro ponto, o erro no resultado final é menor $(<5 \%)$ no local A que no local P (até $15 \%$ ), considerando, dentre outros fatores, o fato de, no local $P$, ter sido utilizada a equação $5 \mathrm{~b}$ e, no local $A$, a $5 a$. Esta última contabiliza a raiz quadrada do val or da leitura $\left(\mathrm{K}_{\mathrm{a}}\right)$ e não o próprio valor como na equação $5 b$.

Salienta-se que todas as avaliações feitas referem-sea comparações com dados experimentais com 25 observações ao longo do tempo da umidade (local P e A) e do potencial matricial (somente no local P). Como todo o processo de cál cul o baseou-se essencial mente em regressões lineares, é óbvio que, por exemplo, um maior número de leituras reduziria o peso que cada uma delas teria no resultado dessas regressões e no resultado final da rotina de cál culo. Dessa forma, a exclusão de uma das observações ou um erro em um dos valores de leitura doTDR teria um efeito tanto menor quanto maior fosse o número total de observações. Sendo assim, fica evidenciada a importância deum númerosuficientedeobservações para evitar que erros ao acaso possam ter influência significativa noresultado final. Erros ao acaso podem tanto subestimar ou superestimar e, como dentro de um conjunto de observações grande haverá um número grandedeerros aoacaso, aumenta a probabilidade de estes se eliminarem no resultado final.

Analisando os erros do processamento de todas as observações, como o término antecipado do experimento ou a calibração doTDR, verificou-se o efeito desses erros sobre o resultado final independe do número de observações realizadas. 

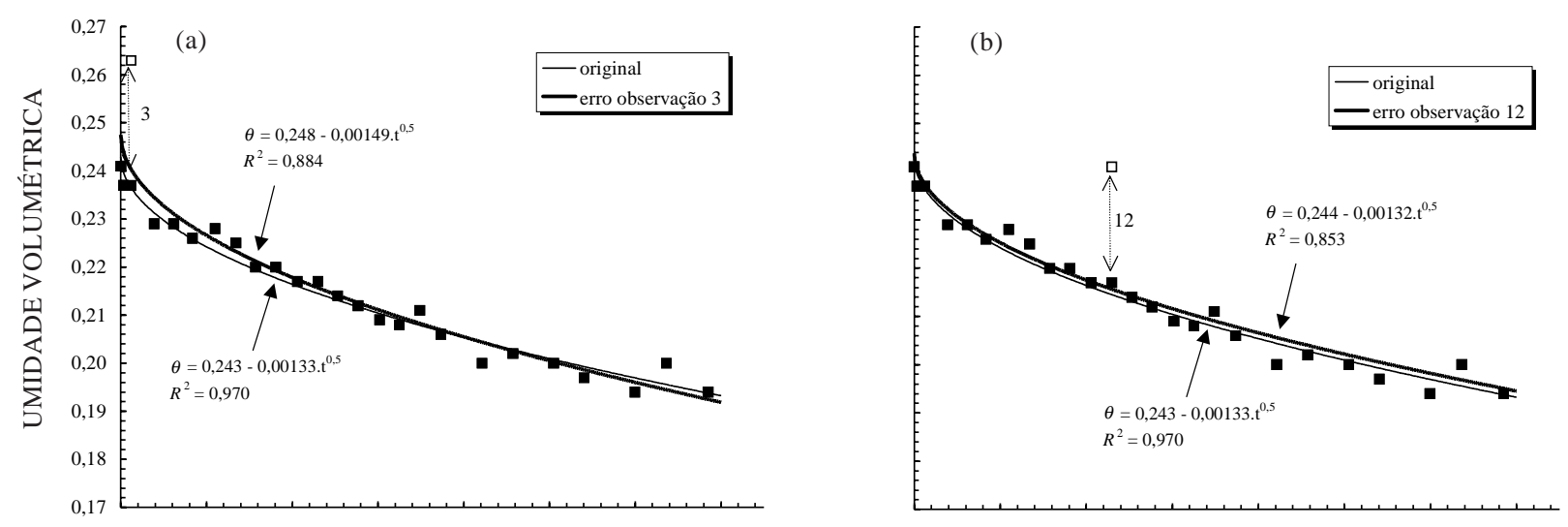

TEMPO, h

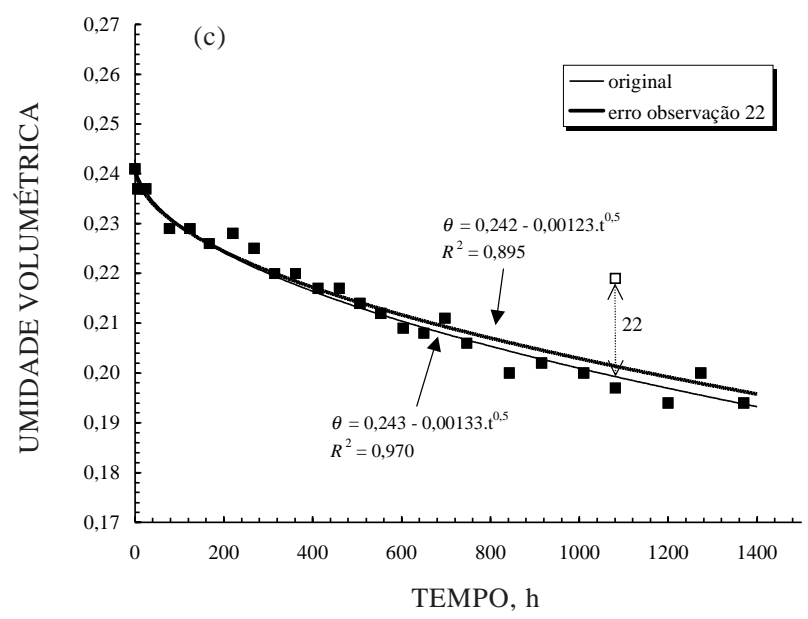

Figura 10. Efeito de um erro de $+10 \%$ em uma das leituras do TDR no local $P(0,30-0,45 \mathrm{~m})$ no ajuste de equação aos valores da umi dade em função do tempo: (a) erro na observação com número de ordem 3; (b) erro na observação com número de ordem 12; (c) erro na observação com número de ordem 22.

\section{CONCLUSÕES}

1. O método do perfil instantâneo, conjuntamente com a rotina computacional utilizada para processar os valores observados no campo, apresenta alta sensibilidade a pequenas incertezas no ajuste de funções aos val ores da umi dade versus tempo.

2. A leitura de tempo zero, ou muito próximo a zero, influencia muito o ajuste de funções aos val ores da umidade versus tempo, razão por que deve ser desconsiderada.

3. Erros ou incertezas nos ajustes dos valores do potencial total versus a profundidadeafetam apenas poucos valores de condutividade hidráulica calculados, tendo assim uma menor influência no resultado final.

4. O comportamento nãoestritamenteexponencial entre condutividade hidráulica e umidade, quando ocorre na faixa de umidades avaliada, aumenta a sensibilidade do método para pequenas alterações no conjunto de dados de entrada.
5. Erros no coeficiente angular e no intercepto, de umTDR com equação de cali ibraçãolinear afetam respectivamente, apenas o coeficiente angular $(\gamma)$ ou o intercepto $\left(\ln \left(K^{*}\right)\right)$, da relação entre condutividade hidráulica e umidade, quando o potencial matricial é determinado por tensiometria.

\section{AGRADE CIMENTOS}

O autor agradece à Fundação de Amparo de Pesquisa do estado de São Paulo (FAPESP), pelo apoi o financeiro, sem o qual os dados experimentais utilizados nesta tese não poderiam ter sido obtidos. Agradece também aos alunos de Doutorado do Programa de Pós-Graduação em Solos e Nutrição de Plantas da ESALQ/USP, Luciana Gomes Castro e J osé Fernandes de Melo Filho, pelo auxílio na execu ção de grande parte do experimento de campo, respectivamente no "Local A" (Fazenda Areão) e "Local P" (Campus ESALQ). 


\section{LITERATURA CITADA}

ANDREU, L.; HOPMANS, J.W. \& SCHWANKL, L.J . Spatial and temporal distribution of soil water balance for a dripirrigated al mond tree. Agric. Water Manag., 35:123-146, 1997.

BOUMA, J .; J ONGMANS, A. \& STEIN, A. Characterizing spatially variable hydraulic properties of a boulder clay deposit in the Netherlands. Geoderma, 45:19-29, 1989.

CHANASYK, D.S. \& NAETH, M.A. Field measurements of soil moisture using neutron probes. Can. J. Soil Sci., 76:317323, 1996.

COMEGNA V.; DAMIANI, P.; D'ANNA, F. \& RUGGIERO, C. Comparison of different field methods for determining the hydraulic conductivity curve of a volcanic Vesuvian soil. Geoderma, 73:231-244, 1996.

DASBERG, S. \& HOPMANS, J.W. Time domain reflectometry calibration for uniformly and nonuniformly wetted sandy and clayey loam soils. Soil Sci. Soc. Am. J., 56:1341-1345, 1992.

DRECHSLER, M. Sensitivity analysis of complex models. Biol. Conser., 86: 401-412, 1998.

HAVERKAMP, R.; VAUCLIN, M \& \& VACHAUD, G. Error analysis in estimating soil water content from neutron probe measurements:1. Local standpoint. Soil Sci., 137:78-90, 1984.

HILLEL, D.A.; KRENTOS, V.K. \& STILIANOV, Y. Procedure and test of an internal drainage method for measuring soil hydraulic characteristics in situ. Soil Sci., 114:395-400, 1972.

HOOK, W.R. \& LIVINGSTON, N.J. Errors in converting time domain reflectometry measurements of propagation velocity to estimates of soil water content. Soil Sci. Soc. Am. J., 60:35-41, 1996

J ONG VAN LIER, Q. \& LIBARDI, P.L. Variabilidade dos parâmetros da equação que relaciona a condutividade hidráulica com a umidade do solo no método do perfil instantâneo. R. Bras. Ci. Solo, 23:1005-1014, 1999.

KLEIN, V.A. Propriedades físico-hídrico-mecânicas de um Latossolo Roxo, sob diferentes sistemas de uso e manejo.Piracicaba, Escola Superior de Agricultura "Luiz de Queiroz", 150p. 1998. (Tese de Doutorado)

LIBARDI, P.L.; REICHARDT, K.; NIELSEN, D.R. \& BIGGAR, J.W. Simplified field methods for estimating the unsaturated hydraulic conductivity. Soil Sci. Soc. Am. J., 44:3-6, 1980.
LOGSDON, S.D. \& J AYNES, D.B. Spatial variability of hydraulic conductivity in a cultivated field at different times. Soil Sci. Soc. Am. J., 60:703-709, 1996.

MARION, J.M.; OR, D.; ROLSTON, D.E.; KAVVAS, M.L.; BIGGAR, J.W. Evaluation of methods for determining soilwater retentivity and unsaturated hydraulic conductivity. Soil Sci., 158:1-13, 1994.

PONIZOVSKY, A.A.; CHUDINOVA, S.M. \& PACHEPSKY, Y.A. Performance of TDR calibration models as affected by soil texture. J. Hydrol., 218:35-43, 1999.

PREVEDELLO, C.L.; KOBIYAMA, M.; JACOBS, G.A. \& DIVARDIN, C.R. Comparação dos métodos do perfil instantâneo e de Van Genuchten na obtenção da condutividade hidráulica de uma areia marinha. R. Bras. Ci. Solo, 19:1-5, 1995.

REICHARDT, K.; PORTEZAN, O.; LIBARDI, P.L.; BACCHI, O.O.S.; MORAES, S.O.; OLIVEIRA, J.C.M.; FALLEIROS, M.C. Critical analysis of the field determination of soil hydraulic conductivity functions using the flux gradiente approach. Soil Till. Res., 48:81-89, 1998.

SALTELLI, A. \& SCOTT, M. The role of sensitivity analysis in the corroboration of models and its link to model structural and parametric uncertainty. Rel. Eng. System Safety, 57:14, 1997.

SIMUNEK, J.; van GENUCHTEN, M.T.; GRIBB, M.M. \& HOPMANS, J. Parameter estimation of unsaturated soil hydraulic properties from transient flow processes. Soil Till. Res., 47:27-36, 1998.

TOPP, G.C.; DAVIS, J.L. \& ANNAN, A.P. Electromagnetic determination of soil water content: measurement in coaxial transmission lines. Water Res. Res., 16:574-582, 1980.

van GENUCHTEN, M.T. A closed-form equation for predicting the hydraulic conductivity of unsaturated soils. Soil Sci. Soc. Am. J., 44:892-897, 1980.

VIEIRA, S.R.; NIELSEN, D.R. \& BIGGAR, J.W. Spatial variability of field-measured infiltration rate. Soil Sci. Soc. Am. J., 45:1040-1048, 1981.

WARRICK, A.W. \& NIELSEN, D.R. Spatial variability of soil physical properties in the field. In: HILLEL, D., ed. Applications of soil physics. New York, Academic Press, 1980. p.319-344.

WATSON, K.K. An instantaneous profilemethod for determining the hydraulic conductivity of unsaturated porous materials. Water Res. Res., 2:709-715, 1966. 
